\title{
Experimental characterisation of light emission during shock-driven cavity collapse
}

Phillip A. Anderson, Nicholas Hawker, Matthew Betney, Brett Tully, Yiannis Ventikos, and Ronald A. Roy

Citation: Proc. Mtgs. Acoust. 19, 075039 (2013); doi: 10.1121/1.4800761

View online: https://doi.org/10.1121/1.4800761

View Table of Contents: http://asa.scitation.org/toc/pma/19/1

Published by the Acoustical Society of America

\section{Articles you may be interested in}

Computational modelling of the interaction of shock waves with multiple gas-filled bubbles in a liquid

Physics of Fluids 27, 036101 (2015); 10.1063/1.4914133

Simulation of warm dense matter in intense bubble collapse

Proceedings of Meetings on Acoustics 19, 075040 (2013); 10.1121/1.4800760

Numerical investigation of collapsing cavity arrays

Physics of Fluids 24, 052104 (2012); 10.1063/1.4719142

Simulation of bubble expansion and collapse in the vicinity of a free surface

Physics of Fluids 28, 052103 (2016); 10.1063/1.4949354

Characterizing shock waves in hydrogel using high speed imaging and a fiber-optic probe hydrophone Physics of Fluids 29, 057101 (2017); 10.1063/1.4982062

Sonoluminescence and bubble dynamics for a single, stable, cavitation bubble

The Journal of the Acoustical Society of America 91, 3166 (1992); 10.1121/1.402855 


\title{
Proceedings of Meetings on Acoustics
}
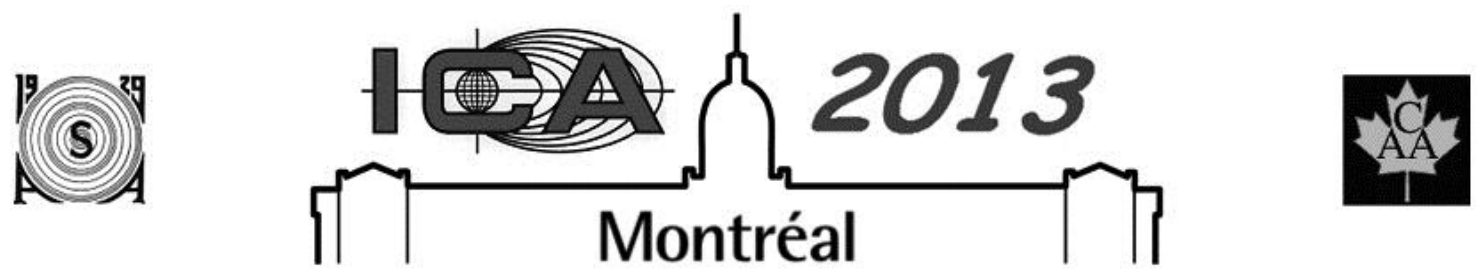

\author{
ICA 2013 Montreal \\ Montreal, Canada
}

2 - 7 June 2013

Biomedical Acoustics

Session 2pBAa: Bubbles Bubbles Everywhere II

\section{2pBAa3. Experimental characterisation of light emission during shock-driven cavity collapse}

Phillip A. Anderson, Nicholas Hawker*, Matthew Betney, Brett Tully, Yiannis Ventikos and Ronald A. Roy

*Corresponding author's address: Department of Engineering Science, University of Oxford, Oxford, OX1 3PJ, Oxfordshire, United Kingdom, nicholas.hawker@eng.ox.ac.uk

The authors describe experimental work examining the collapse of a cavity by a strong shockwave. A millimetre size cavity is cast in Phytagel, which is then impacted by a metallic projectile accelerated by a compressed gas gun, reaching velocities up to $500 \mathrm{~m} / \mathrm{s}$. The impact generates a strong shockwave that propagates into the gel at greater than sonic velocity. Schlieren images are presented that illustrate both this process and the subsequent cavity collapse at a sub-microsecond timescale. As the shockwave reaches the cavity it is shown to cause a rapid asymmetric collapse process characterised by the formation of a high-speed transverse jet. The pressure of the shockwave is found to be 100+ MPa as measured via a custom-built fibre-optic probe hydrophone. Previous work examining shock-driven cavity collapse observed luminescence, postulated to be due to the high-speed impact of the transverse jet on the far bubble wall; this experimental observation is replicated. Further, the light emission is characterised as a function of impact velocity and thus of shockwave pressure. This reveals that shock-driven cavity collapse shares many of the unique features that make the more widely studied SBSL-type collapse interesting.

Published by the Acoustical Society of America through the American Institute of Physics 


\section{INTRODUCTION}

Bubble collapse has been a subject of great interest ever since it was connected with the erosion of ship propellers a century ago [1]. Since that time, much effort has been made to understand this process in order to mitigate or harness its effects.

The simplest and best understood case is the symmetric collapse of a single bubble. This problem has been studied extensively, aided in particular by a simplified theoretical treatment $[1,2,3]$ and the development of acoustic levitation techniques [4, 5]. Single bubble dynamics are a stepping stone to understanding more complex bubble phenomena, but historically much interest has been fueled by sonoluminescence (SL), the phenomenon of light emission from a collapsing bubble. Measurements of SL have proven to be crucial for gaining insight into the extreme conditions present inside the collapsing bubble and have led to speculation of more exotic bubble applications [6, 7].

Asymmetric collapse is also well studied, particularly in the context of bubbles near boundaries $[8,9,10,11,12]$. This is often in pursuit of damage mitigation methods as applied to ships, hydraulic equipment, biological systems, and so on. The presence of a boundary near the bubble inhibits symmetric forcing of the interface, and often results in the formation of a high-velocity liquid jet through the bubble. Similar dynamics are observed for shock-induced collapse $[13,14]$. This phenomenon is less well understood, particularly due to the numerical complexity and experimental difficulty of researching such interactions. Apart from numerical treatments, there has been little experimental work done on this problem. One exception is a series of experiments started by Dear et al. in the late 1980s [13, 15, 16, 17, 18, 19, 20].

We know from SL literature that luminescence is a powerful way to gain insight to the extreme conditions inside the bubble. However, despite the observance of luminescence, no attempts have been made to further quantify the light in the case of shock-induced collapse. The current effort aims to recreate and further the experiments of Dear et al. by quantifying light emission from shock-induced bubble collapse. This experimental work will be combined with ongoing numerical work using state-of-the-art front tracking methods [14].

\section{EXPERIMENTAL SETUP}

The idealized problem considers the interaction of a bubble or cavity in a fluid with a planar shockwave. To that end, an experimental apparatus similar to that used in the work of Bourne and Field has been reproduced. The setup consists of a disposable gel target placed at the end of a compressed gas gun. The target is enclosed in a large metal box to prevent debris from damaging sensitive diagnostic equipment.

The severe challenges of creating and placing a bubble in a fluid such as water are bypassed entirely by instead casting a cavity in gel. The cavity is thus 'frozen' in space and easily reproduced. Although the gel is solid under normal conditions, it mimics water under the extreme conditions presented by a shockwave [21]. Use of a gel will also be beneficial for future work, where more complex geometries may be desirable. Gellan gum (Phytagel ${ }^{\mathrm{TM}}$, Sigma-Aldrich) was chosen for its optical clarity and ease of handling.

For this work we consider a single cylindrical cavity as a baseline geometry. Gel targets are created by casting three $50 \mathrm{~mm} \times 50 \mathrm{~mm}$ slabs. The central slab is $10 \mathrm{~mm}$ thick with a $5 \mathrm{~mm}$ diameter cylindrical cavity cast in the center. This slab is then sandwiched between two solid $20 \mathrm{~mm}$ side slabs. The gel is assembled on a PVC base with an aluminum striker, as shown on the left of figure 1. The striker is set to overhang the PVC base to compensate for differences in sound speed between PVC and the gel material.

The target assembly is mounted at the end of a $70 \mathrm{~mm}$-bore single-stage compressed-gas 


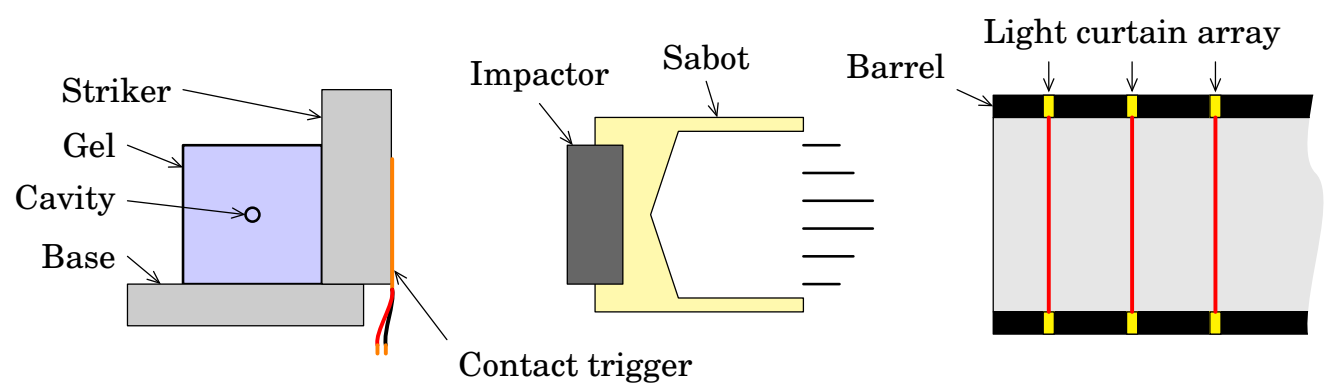

FIGURE 1: Side schematic of the impact. Steel projectile traveling to the left impacts the aluminum striker. Shockwave enters the gel and forces the cavity to collapse. Projectile velocity is calculated from the light curtains. Time of impact is given by the contact trigger. The projectile and target assembly are disposable.

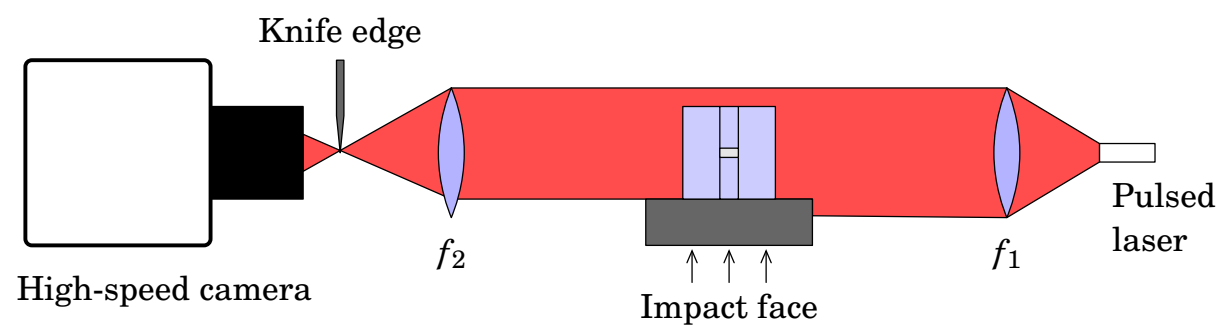

FIGURE 2: Schematic of the schlieren setup from above. Illumination is provided by a pulsed diode laser coupled into a liquid light guide. Field lenses $\left(f_{1}\right.$ and $\left.f_{2}\right)$ are $76 \mathrm{~mm}$ diameter with a focal length of $850 \mathrm{~mm}$. The high-speed camera is focused on the center of the gel. A protective box (not shown) shields the equipment from impact debris.

gun. When a projectile fired from this gun impacts the striker it generates a shockwave which propagates into the gel. The aluminum striker plate thus serves two purposes. It protects the gel from airflow before impact and acts as an acoustic matching layer. Our projectile consists of a $50 \mathrm{~mm}$ diameter mild steel disk impactor set inside a nylon sabot (figure 1 center). Total mass of the projectile is around $360 \mathrm{~g}$. Even at this weight, speeds greater than $500 \mathrm{~m} / \mathrm{s}$ are achievable.

Projectile velocity is calculated with timings obtained from a light curtain array (LCA) at the end of the gun barrel (figure 1 right). Although it is well suited for velocity measurement, the LCA is not precise enough to trigger diagnostic devices. A more robust trigger is required to capture the event of interest, which occurs within $30 \mu \mathrm{s}$ of impact. Thus, triggering of diagnostic equipment is achieved with a Schmidt trigger attached to two parallel copper strips mounted on the impact face of the striker. Contact with the steel impactor bridges the strips and triggers data acquisition.

One of the primary diagnostics is a high-speed camera (SimX-16, Specialised Imaging) capable of acquiring sixteen $1280 \times 960 \mathrm{px}^{2}$ images at frame rates up to $200 \mathrm{Mfps}$. This camera is used to capture the collapse event in two different configurations, as shown in figures 2 and 3. The first configuration (figure 2) is a standard schlieren setup for imaging optical inhomogeneities [22]. Schlieren is particularly useful for observing the passage of the shockwave past the cavity. Images are back-lit using a $200 \mathrm{~W}$ pulsed diode laser with a $25 \mu \mathrm{s}$ maximum pulse width and $640 \mathrm{~nm}$ center wavelength (Cavilux SMART, Cavitar Ltd.). Both field lenses have a diameter of $76 \mathrm{~mm}$ and a focal length $f=850 \mathrm{~mm}$.

The second configuration (figure 3 ) is used to observe luminescence from cavity collapse itself. The pulsed laser is not used in this configuration as it would wash out contributions from the collapse. Additionally, the knife edge is removed to maximize light collection. Since the laser 


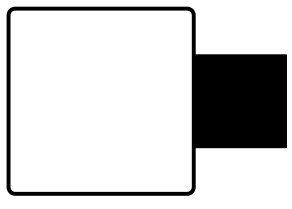

High-speed camera

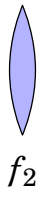

$f_{2}$

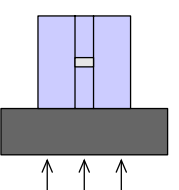

Impact face

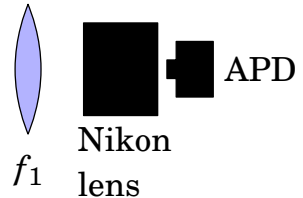

$f_{1}$ lens

FIGURE 3: Schematic of the APD setup from above. The pulsed laser is removed and replaced by a lens and APD. The knife edge is also removed. The gain of the high-speed camera is increased so that it is sensitive enough to capture the light emitted during collapse of the cavity.

is no longer needed, the high-speed images can be supplemented with a fast light detector to collect temporal data. For this purpose, an avalanche photo-detector (APD) is used. A $50 \mathrm{~mm}$, $f 1.8$ Nikon lens is placed behind the field lens and adjusted to focus the region around the cavity on the $1 \mathrm{~mm}$ diameter sensing element of the APD (APD110A2/M, Thorlabs). In practice, it is straightforward to switch between these two configurations with minimal realignment of optics.

The final diagnostic is a fiber-optic probe hydrophone (foph) which was built following the design of Parsons et al. [23, 24]. Calibration was performed using calibrated oils (AAA-1/2 Series and selected others, Cargille Labs), as described by Arvengas [25]. The probe tip is inserted from the top of the gel to the desired depth. In this way, the shape and amplitude of the shockwave can be measured. The fiber end is destroyed each shot and hence must be re-cleaved for every use.

\section{RESULTS}

Figure 4 shows a series of schlieren images from a single event. The shockwave generated by the impact propagates into the gel towards the cavity from the right side. Several features typical of shock-induced collapse are visible in the images. First, a rarefaction fan is emitted as the shock passes over the cavity (figure 4, frames 6-11). As the cavity collapses, the buckling interface forms a jet (figure 4, frame 9) which impacts the leeward side. The resulting water hammer shock from this impact appears in figure 4 frames 11-16.

The treatment of this collapse process is well established in both numerical and experimental work. The asymmetric forcing of the cavity interface results in an asymmetric collapse, as illustrated in figure 5. Following the jet impact, the collapse of the remaining lobes of the cavity leads to the formation of hot spots, which may luminesce.

With the backlighting removed, luminescence from the cavity collapse can be imaged, as shown in figure 6. A back-lit image (figure 6a) is taken beforehand as a reference. The backlight is then removed and the camera gain is increased to maximize sensitivity. Two distinct hotspots are clearly visible (figure 6c), and an overlay of the two images (figure $6 \mathrm{~b}$ ) reveals that the flashes are located approximately where lobes are expected to form.

Due to the brief duration of the flash, on the order of $300 \mathrm{~ns}$, and the uncertainty about when it will occur, the camera is set to take $1.75 \mu$ s exposures. The high-speed images therefore reveal very little temporal information. APD measurements overcome this deficiency by providing temporal information at the cost of spatial resolution. Figure 7 compares the APD output from two collapse events with different shock amplitudes. While the flash duration in both cases is similar, the shock generated by the $461 \mathrm{~m} / \mathrm{s}$ impact yields 6 times the peak power compared to a $276 \mathrm{~m} / \mathrm{s}$ impact. 


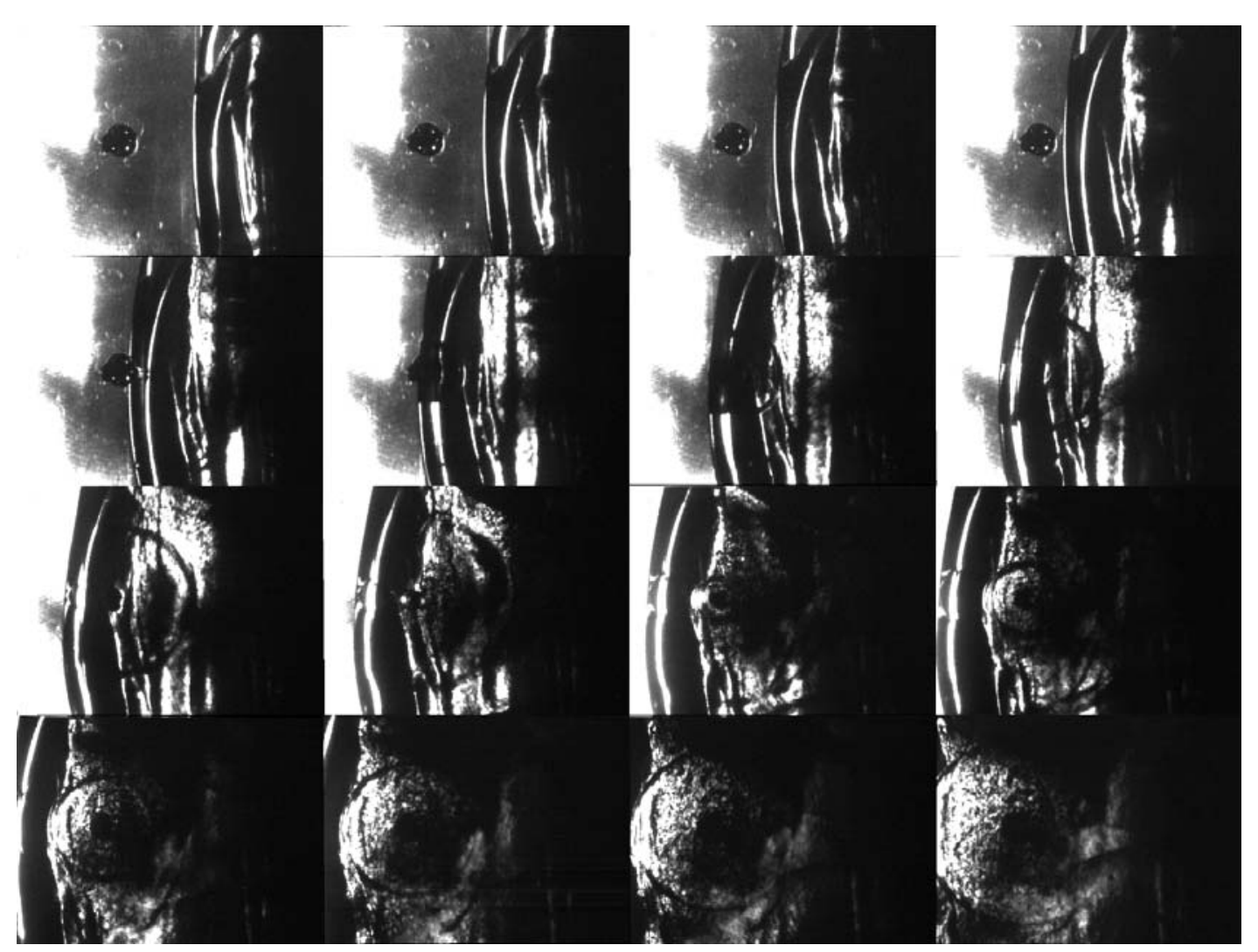

FiguRE 4: Schlieren images of a collapse event. The cavity is $5 \mathrm{~mm}$ in diameter and $10 \mathrm{~mm}$ long. The shockwave enters from the right edge of the image. Interframe time is $1.5 \mu \mathrm{s}$ and progression is left to right, top to bottom.

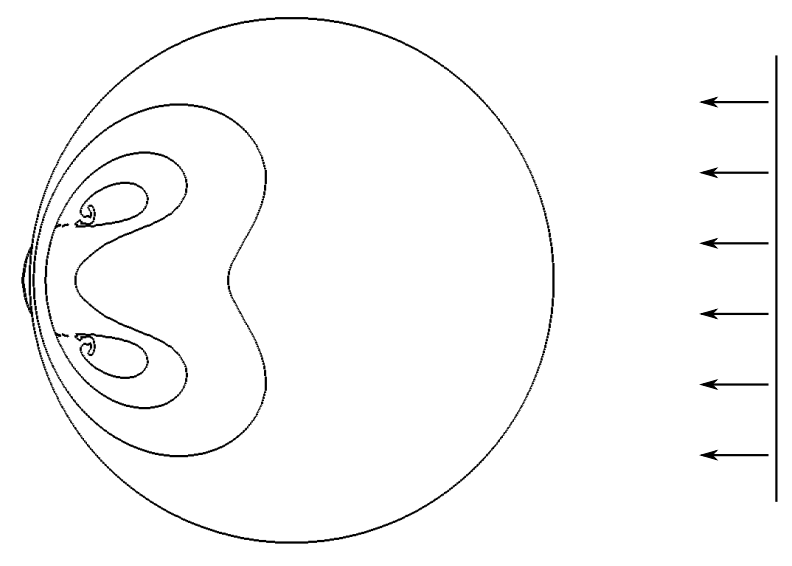

FIGURE 5: Simulated asymmetric collapse of a 2D cavity from a $250 \mathrm{MPa}$ planar shockwave. Shockwave enters from the right side. The bubble interface involutes and forms a jet which impacts the leeward side. The remaining lobes are compressed and subsequently luminesce. 


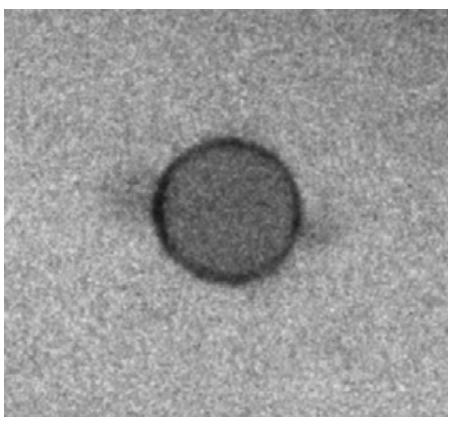

(A)

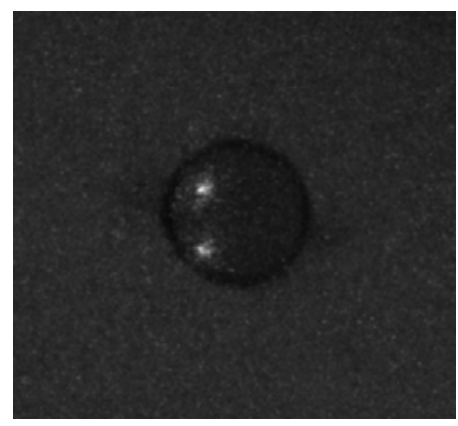

(B)

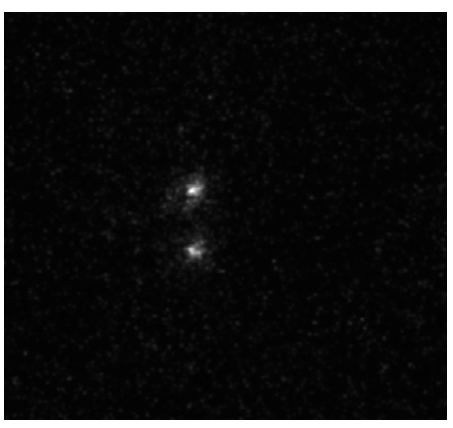

(C)

FIGURE 6: (A) Backlit reference image of the cavity. The cavity diameter is $5 \mathrm{~mm}$. (B) Superimposed image. (C) Luminescence from the cavity collapse.

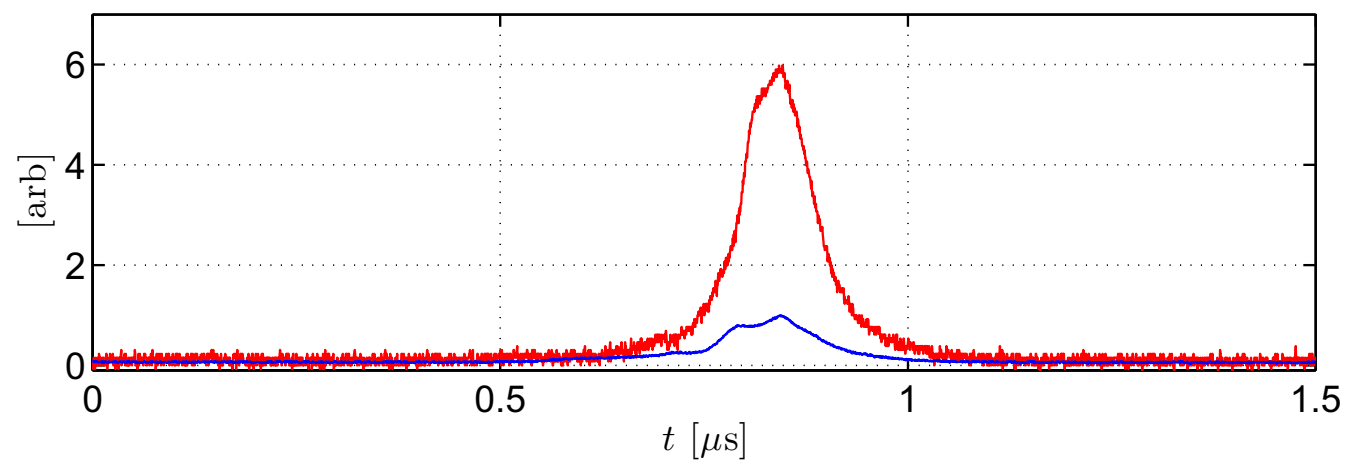

FIGURE 7: Sample traces from the APD, normalized by the lower peak. The lower trace (blue) corresponds to a collapse from a $276 \mathrm{~m} / \mathrm{s}$ impact, and the upper trace (red) from a $461 \mathrm{~m} / \mathrm{s}$ impact. Peaks have been artificially aligned for comparison. 


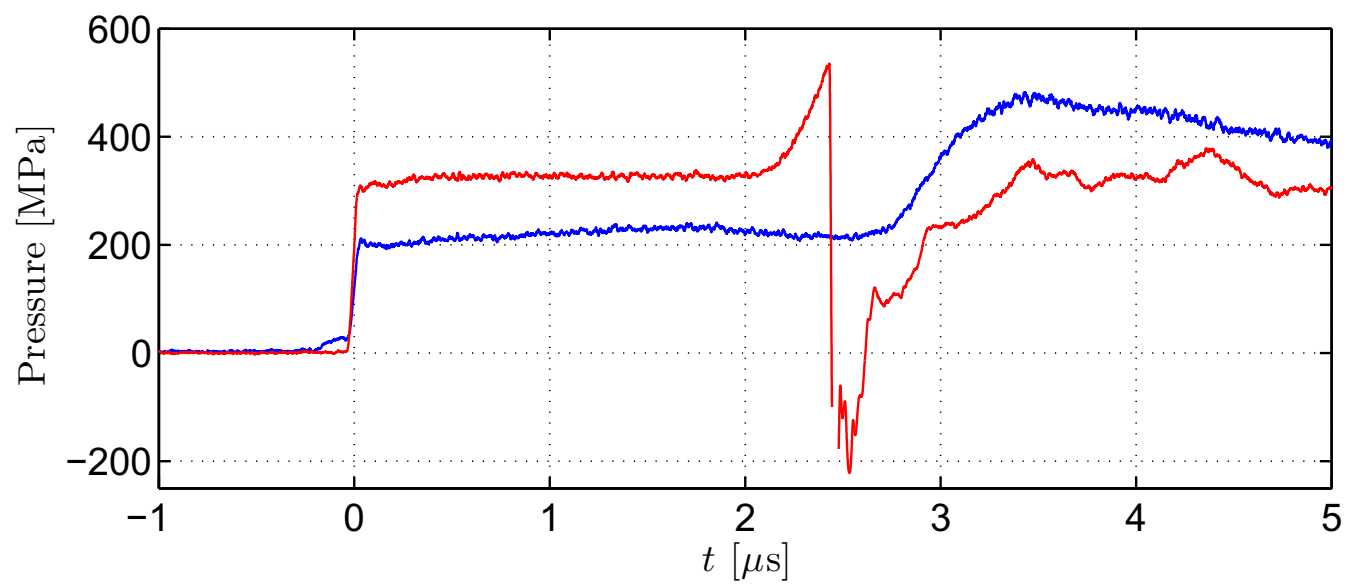

FIGURE 8: Sample traces from the foph. The lower trace (blue) corresponds to the shockwave from a $291 \mathrm{~m} / \mathrm{s}$ impact, and the upper trace (red) from a $445 \mathrm{~m} / \mathrm{s}$ impact. Rising edges have been artificially aligned for comparison. The discontinuity near $2.5 \mu \mathrm{s}$ is a result of the fiber breaking.

Figure 8 compares two foph traces for impacts at $291 \mathrm{~m} / \mathrm{s}$ and $445 \mathrm{~m} / \mathrm{s}$. In both cases there is a sharp rise followed by a relatively long period of near constant high pressure on the order of several microseconds. Unfortunately, interpretation of the foph traces at later times is hindered by uncertainty in the integrity of the fiber. The peak amplitudes agree reasonably well with predictions based on a linear three-medium problem.

The work presented here shows first steps towards characterizing luminescence from shock-induced collapse. Further experiments will characterize the shockwave amplitude as a function of the projectile velocity. Combined with luminescence measurements, this will allow for comparison with current numerical efforts. 


\section{REFERENCES}

[1] L. Rayleigh, "On the pressure developed in a liquid during the collapse of a spherical cavity”, Philosophical Magazine 34, 94-98 (1917).

[2] F. R. Gilmore, "The growth or collapse of a spherical bubble in a viscous compressible liquid", Technical Report 26-4, California Institute of Technology Hydrodynamics Laboratory, Pasadena, CA (1952).

[3] R. Hickling and M. S. Plesset, "Collapse and Rebound of a Spherical Bubble in Water", Physics of Fluids 7, 7 (1964).

[4] D. F. Gaitan and L. A. Crum, "Sonoluminescence from single bubbles", Journal of the Acoustical Society of America 87, S141--S141 (1990).

[5] D. F. Gaitan, "An experimental investigation of acoustic cavitation in gaseous liquids", Ph.D. thesis, University of Mississippi (1990).

[6] K. S. Suslick, “Sonochemistry”, Science 247, pp. 1439—-1445 (1990).

[7] W. C. Moss, D. B. Clarke, J. W. White, D. A. Young, and W. C. M. I, "Sonoluminescence and the prospects for table-top micro-thermonuclear fusion”, Physics Letters A 211, 69-74 (1996).

[8] W. Lauterborn and H. Bolle, "Experimental investigations of cavitation-bubble collapse in the neighbourhood of a solid boundary", Journal of Fluid Mechanics 72, 391--399 (1975).

[9] D. C. Gibson and J. R. Blake, "The growth and collapse of bubbles near deformable surfaces", Applied Scientific Research 38, 215-224 (1982).

[10] T. Kodama and Y. Tomita, "Cavitation bubble behavior and bubble-shock wave interaction near a gelatin surface as a study of in vivo bubble dynamics", Applied Physics B: Lasers and Optics 70, 139-149 (2000).

[11] E. Johnsen and T. Colonius, "Numerical simulations of non-spherical bubble collapse", Journal of Fluid Mechanics 629, 231-262 (2009).

[12] P. V. Chitnis, N. J. Manzi, R. O. Cleveland, R. a. Roy, and R. G. Holt, "Mitigation of Damage to Solid Surfaces From the Collapse of Cavitation Bubble Clouds", Journal of Fluids Engineering 132, 51303 (2010).

[13] J. P. Dear, J. E. Field, and A. J. Walton, "Gas compression and jet formation in cavities collapsed by a shock wave”, Nature 332, 505-508 (1988).

[14] N. A. Hawker and Y. Ventikos, "Interaction of a strong shockwave with a gas bubble in a liquid medium: a numerical study", Journal of Fluid Mechanics 701, 59-97 (2012).

[15] N. K. Bourne and J. E. Field, "Bubble Collapse and the Initiation of Explosion", Proceedings of the Royal Society A: Mathematical, Physical and Engineering Sciences 435, 423-435 (1991).

[16] N. K. Bourne and J. E. Field, "Shock-induced collapse of single cavities in liquids", Journal of Fluid Mechanics 244, 225-240 (1992).

[17] N. K. Bourne and J. E. Field, "Shock-induced collapse and luminescence by cavities", Philosophical Transactions of the Royal Society A: Mathematical, Physical and Engineering Sciences 357, 295-311 (1999). 
[18] N. K. Bourne and J. E. Field, "Explosive ignition by the collapse of cavities", Proceedings of the Royal Society A: Mathematical, Physical and Engineering Sciences 455, 2411-2426 (1999).

[19] N. K. Bourne, “On the collapse of cavities”, Shock Waves 11, 447-455 (2002).

[20] N. K. Bourne and A. M. Milne, "The temperature of a shock-collapsed cavity", Proceedings of the Royal Society A: Mathematical, Physical and Engineering Sciences 459, 1851-1861 (2003).

[21] J. E. Field, J. P. Dear, and J. E. Ogren, "The effects of target compliance on liquid drop impact”, Journal of Applied Physics 65, 533-540 (1989).

[22] G. S. Settles, Schlieren and Shadowgraph Techniques (2001).

[23] J. Staudenraus and W. Eisenmenger, "Fibre-optic probe hydrophone for ultrasonic and shock-wave measurements in water", Ultrasonics 31, 267-273 (1993).

[24] J. E. Parsons, C. A. Cain, and J. B. Fowlkes, "Cost-effective assembly of a basic fiber-optic hydrophone for measurement of high-amplitude therapeutic ultrasound fields", Journal of the Acoustical Society of America 119, 1432-1440 (2006).

[25] A. Arvengas, K. Davitt, and F. Caupin, "Fiber optic probe hydrophone for the study of acoustic cavitation in water.", The Review of scientific instruments 82, 034904 (2011). 